\title{
Non-functioning Pancreatic Neuroendocrine Tumor in a Patient with Multiple Endocrine Neoplasia Type 1 Syndrome: Case Report
}

\author{
Gabriel Brito Barbosa ${ }^{1, *}$, Lucas Santos Pimentel ${ }^{1}$, Arthur Ribeiro Costa Carvalho ${ }^{2}$, \\ Thais Ribeiro Costa Carvalho ${ }^{3}$, Ana Rita Marinho Ribeiro Carvalho ${ }^{4}$ \\ ${ }^{1}$ Centro Universitário Maurício de Nassau (UNINASSAU), Brazil \\ ${ }^{2}$ Faculdade de Medicina de Olinda (FMO), Brazil \\ ${ }^{3}$ Faculdade Pernambucana de Saúde (FPS), Brazil \\ ${ }^{4}$ Brazilian College of Radiologists, Brazil
}

Copyright $\mathrm{C} 2019$ by authors, all rights reserved. Authors agree that this article remains permanently open access under the terms of the Creative Commons Attribution License 4.0 International License

\begin{abstract}
Background: Neuroendocrine tumors are rare, but when coexisting with Multiple Endocrine Neoplasia Type 1 Syndrome in the same patient, the literature shows 80 to $100 \%$ association. Represents an autosomal dominant disorder due to mutations in tumor suppressor gene MEN1 and it's defined as the development of two or more tumors from the main three endocrine sites: the parathyroid glands, the pancreatic islet and the Pituitary gland. We present a case of a patient with a compatible family history and classic imaging findings for MEN1 Syndrome. Case report: Male, 28 years old patient genetically diagnosed with the MEN 1 Syndrome who presented to our image service for abdominal tumors' screening. Submitted through multiples imaging exams such as CT, MRI, PET/CT to follow up the diagnosis, in which was found a pancreatic mass measuring over $3 \mathrm{~cm}$, with another 3 little pancreatic lesions as well. The patient received indication to surgery, with further anatomopathological and immunohistochemistry studies confirming the well- differentiated neuroendocrine tumor. Conclusion: Due to the wide range of malignant potential, it's recommended annual screening, as minimum, with biochemical and image techniques. Surgery is the only treatment capable of tumor's eradication, which consists on a pancreatectomy (lesions sized more than 1-2 cm).
\end{abstract}

Keywords Multiple Endocrine Neoplasia Type 1, Neuroendocrine Tumors, Radiology, Computerized Tomography, Magnetic Resonance Image, Endoscopic Ultrasound

\section{Introduction}

Multiple Endocrine Neoplasia Type 1 Syndrome (MEN1) is an autosomal dominant disorder due to mutations in tumor suppressor gene $M E N 1$, which encodes a 610-amino acid protein, menin. First degree relatives have a $50 \%$ risk of developing the disease [1].

Pancreatic Neuroendocrine Tumors (PNETs) are rare (less than $5 \%$ of pancreatic tumors) heterogeneous group of neoplasms that arise from progenitor islet cells but when associated to MEN 1 syndrome, $80-100 \%$ of patients develop them [2]. Also, they commonly occur in younger patients (less than 40 years) when compared to patients without the syndrome, and they can be multiple. $95 \%$ of patients also present primary hyperparathyroidism [1].

Almost all patients with MEN1 will develop a PNET during their lifetime ( $>95 \%$ ), although most of them will be non-functioning micro-adenomas (smaller than $0.5 \mathrm{~cm}$ ) that are typically multifocal. Non-functional PNETs (NF-PNETS) represent $60-90 \%$ of patients and they commonly present later with general symptoms such as abdominal pain, weight loss, anorexia and nausea [2].

We present a 28 years old male patient with family history and genetic diagnose of MEN 1 syndrome who was diagnosed with a NF-PNET. Since approximately all NF-PNETs are diagnosed in advanced stages as they don't cause any specific hormonal symptom (in some cases even with metastatic disease) an early diagnose is crucial and here the radiology has a fundamental role on it.

\section{Case Report}

J.C.A.M, 28 years, male, genetically diagnosed with Multiple Endocrine Neoplasia Type 1 Syndrome (MEN 1), attends to the image service for abdominal tumors' screening. He has a sister, mother and five uncles also genetically diagnosed with the syndrome. Past of 
nephrolithiasis since 20 years old, presented with a discreet increase in parathyroid hormone (PTH) and also serum calcium and scintigraphy image with parathyroid functional adenomas. Presented slight unspecific abdominal pain.

Patient's Computed Tomography (CT) showed a solid lesion in pancreas's head, with heterogenic contrast enhancement, measuring $3.0 \mathrm{~cm}$. Magnetic Ressonance Image (MRI) showed little cystic areas in the lesion, restriction to diffusion in the same spot and in other three milimeterical spots in the pancreatic body (Fig $\mathbf{1}-\mathbf{5})$.

Positron Emission Tomography (PET/CT) with DOTATOC - 68Ga showed mass's increased radiotracer caption (SUVmax - Standardized Uptake Value - 59.4) and in three other focal areas (SUVmax 15.1). The pancreatic lesions were non-functioning, and there were no clinical or laboratory alterations (Fig. 6a-6c).
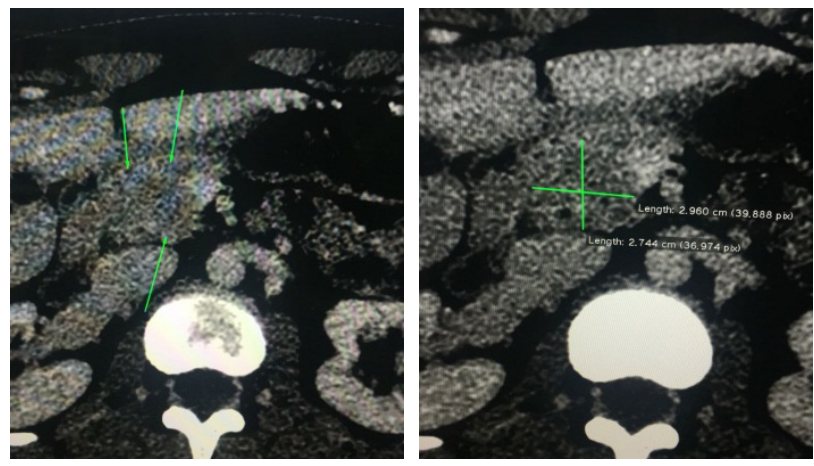

Figure 1a-1b. Portal phase CT shows solid lesion in pancreas's head

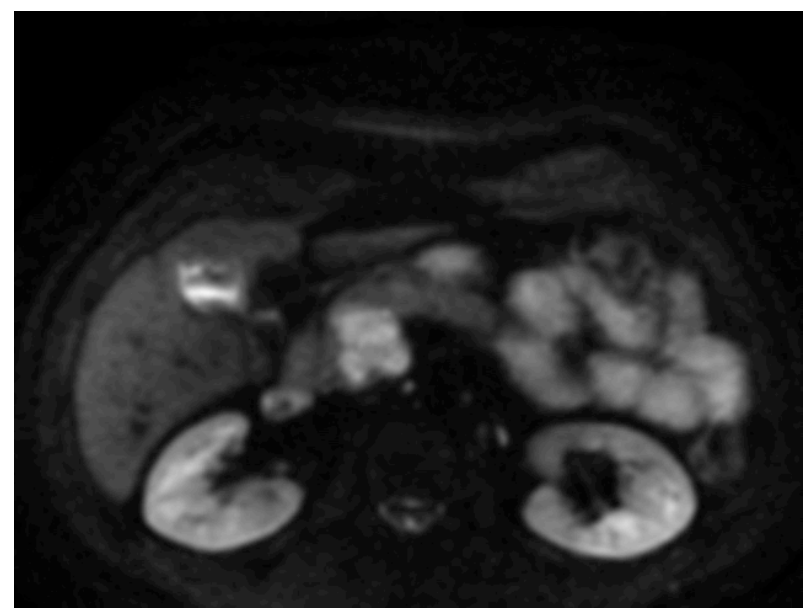

Figure 2. Difusion-weighted MRI showing restrictive lesion located in Pancreas's head

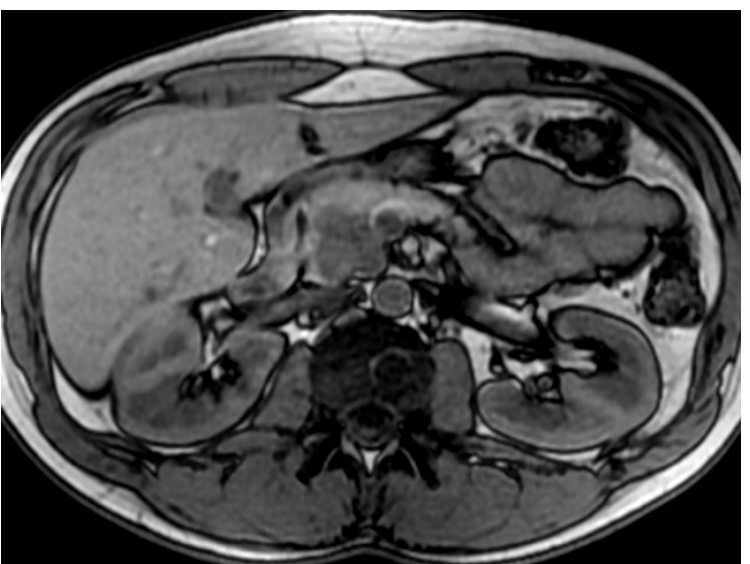

Figure 3. Out-of-Phase T1-weighted GRE MRI showing lobulated image with decreased signal located in Pancreas's head

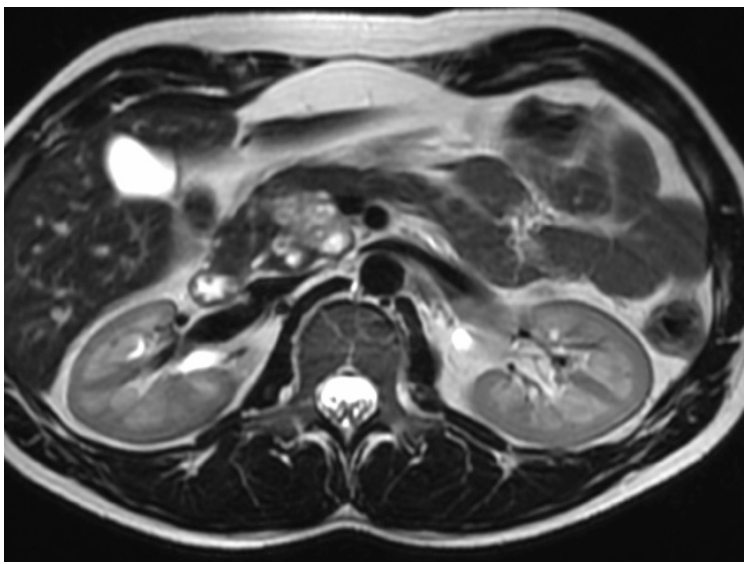

Figure 4. T2-weighted MRI shows hyperintese cystic areas in the lesion

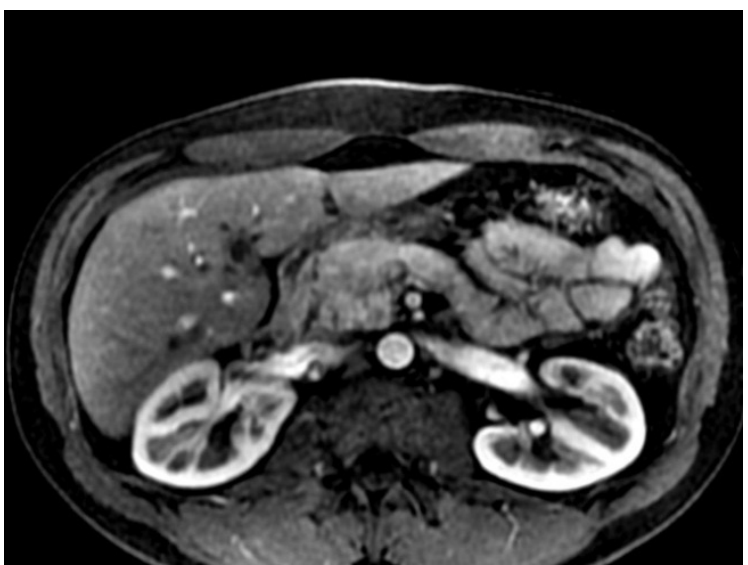

Figure 5. T1-weighted MRI GRE with fat-sat in arterial phase of Gadolinium administration. Observe lesion's heterogenic enhancement 

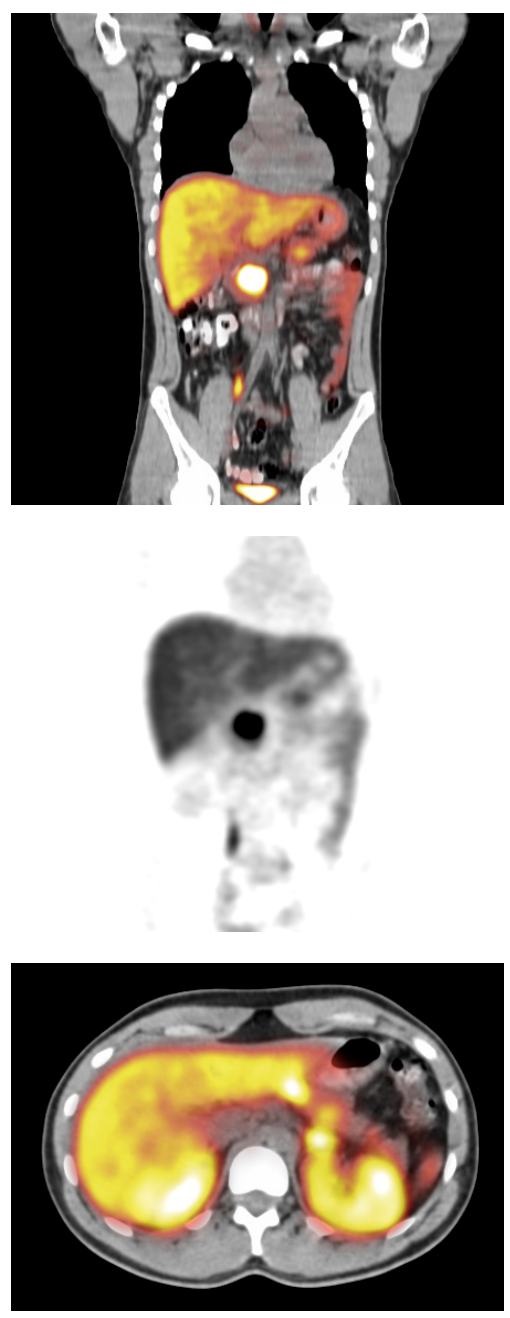

Figure. 6a - 6c. PET/CT with DOTATOC/Ga68 exhibits increased radiotracer caption, greater in Pancreas's head (SUVmax 59,4) but also in three other focal areas.
The patient had a colangiographic Image that demonstrates lesion's cystic component, which didn't communicate with the pancreatic ductal system or determinate biliary ducts dilatation (Fig. 7). Literature shows that tumors measuring less than $2 \mathrm{~cm}$ are usually benign and well-differentiated, justifying surgery indication as the first choice, considering the patient's age and the size of lesion over $3 \mathrm{~cm}$. After resection, the tumor anatomopathological report confirmed a well-differentiated neuroendocrine tumor, grade 1. Also, Immunohistochemistry (IHC) study of the lesion was consistent with grade 1 neuroendocrine tumor, with a proliferative index (Ki-67) of $0,95 \%$ (in WHO 2010 classification) - Table 1.

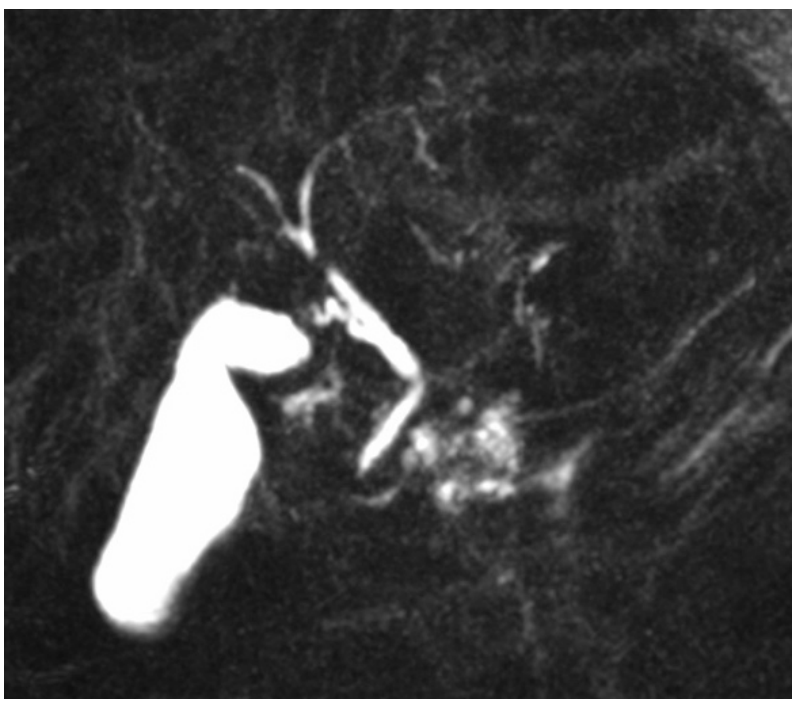

Figure 7. Colangiographic image showing lesion's cystic component. The lesion didn't communicate with pancreatic ductal system or determinate biliary ducts dilatation

Table 1. WHO 2010 Neuroendocrine Tumors Classification

\begin{tabular}{|c|c|c|c|c|}
\hline Differentiation & Grade & Ki-67 Index & Mitotic Count & Nomenclature \\
\hline $\begin{array}{c}\text { Well } \\
\text { Differentiated }\end{array}$ & Low (G1) & $<3 \%$ & $<2$ per 10 HPF & Neuroendocrine Tumor grade 1 \\
\cline { 2 - 5 } & Intermediate (G2) & $3 \%-20 \%$ & $2-20$ per 10 HPF & Neuroendocrine Tumor grade 2 \\
\hline $\begin{array}{c}\text { Poorly } \\
\text { Differentiated }\end{array}$ & High (G3) & $>20 \%$ & $>20$ per 10 HPF & Neuroendocrine Carcinoma \\
\hline
\end{tabular}




\section{Discussion}

MEN 1 is defined as the development of two or more tumors from the main three endocrine sites: the parathyroid glands (almost $100 \%$ of patients presents with Primary Hyperparathyroidism), the pancreas/duodenum/foregut area (termed as Pancreatic Neuroendocrine Tumors PNETs) and the Pituitary glands [1]. As the last one isn't as common as the other two, we won't be discussing it here.

The syndrome is inherited in an autosomal dominant fashion and results from the dysfunction of the protein menin, coded by a gene (MENI) located on chromosome 11. It's expressed and involved in numerous protein-protein interactions regulating cell proliferation and DNA maintenance [3].

Parathyroid adenomas are the most frequently occurring tumor in patients with $M E N 1$, occurring in $90 \%$ of patients by age of 40 . These tumors can cause primary hyperparathyroidism, and patients may present with hypercalcemia, nephrolithiasis, and osteitis fibrosa cystica. The age of onset of symptoms of parathyroid adenoma is earlier in patients with MEN1 than in those without, typically occurring at approximately 20-25 years of age compared to 55 years of age, respectively.

Evaluation for patients with hyperparathyroidism typically includes an ultrasound as an initial screening for underlying parathyroid adenoma followed by nuclear technetium Tc-99m sestamibi scintigraphy for confirmation. Sestamibi scintigraphy has a sensitivity and specificity of $>90 \%$ for lesions that demonstrate retention of sestamibi radiotracer on delayed imaging.

Management of parathyroid adenomas is typically surgical, with removal of the overactive glands with either a subtotal or total parathyroidectomy; however, noting that patients with MEN1 have an increased risk of persistent or recurrent hypercalcemia after subtotal surgery, which is thought to be secondary to their tendency toward multiglandular disease, is important. Biochemical screening for primary hyperparathyroidism in patients with MEN1 should be performed annually with an assessment of serum calcium and parathyroid hormone levels [4].

The PNETs are a rare and heterogeneous group of neoplasms that arise from progenitor islet cells. PNETs may be classified as either functional (F-PNET) or non-functional (NF-PNET), depending on their ability to secrete biologically active hormones and elicit characteristic symptomatology [5].

NF-PNETs, which represents $60-90 \%$ of all PNETs [2], exhibit a wide range of malignant potential, ranging from slow-growing and non-infiltrative tumors to locally invasive and rapidly metastasizing ones. They can remain asymptomatic before they reach a significant tumor burden.

When they become symptomatic, their symptomatology is typically related to mass effect from the primary tumor or the metastasis. As many of them occur in pancreas's head (like in our case), symptoms may include jaundice, abdominal pain, or weight loss.

Other less frequent symptoms may include anorexia, nausea, intra-abdominal bleeding, or a palpable mass. The vast majority of metastasis occurs in the liver [5].

Functional PNETs include insulinomas, gastrinomas, VIPomas and somatostatinomas. The symptoms are due to the hyperproduction of hormones. Insulinomas are the most common F-PNETs and their presentation are resumed in the Whipple triad, which consists of documented hypoglycemia, neuroglycopenic symptoms and rapid reversal of symptoms with administration of glucose [2].

Also, increased insulin resistance appears to be more common in patients with MEN1 than in general population. It has been suggested that this phenomenon (related to a greater cardiovascular risk) may contribute to the increased mortality in MEN1 patients, especially as a considerable number of patients with MEN1 do not die as a direct consequence of their endocrine tumors [3].

In 2012, the American Endocrine Society published a guideline that recommends annual screening, minimally annual plasma biochemical evaluation of a fasting gastrointestinal hormonal profile, which includes measurement of gastrin, glucagon, vasointestinal polypeptide, pancreatic polypeptide, chromogranin A, and insulin with an associated fasting glucose level. Also, a suggested minimum imaging protocol includes annual pancreatic and duodenal visualization with magnetic resonance imaging (MRI), computed tomography (CT), or endoscopic ultrasound (EUS)[1].

In CT, sensibility in the detection of PNETs ranges from 63 to $82 \%$ and specificity ranges between 85 and $100 \%$. It shows lesions extremely vascularized, better evaluated in triphasic dynamic study, mostly showing arterial phase enhancement with portal phase wash-out [2].

MRI is an alternative modality with the advantage of less radiation exposure. PNETs should be low signal intensity on T1 weighted images and high signal intensity on T2 weighted images. In addition, MRI may be more sensitive than $\mathrm{CT}$ for detecting smaller pancreatic lesions and liver metastases [5].

On MRI, in contrast to pancreatic adenocarcinoma, most of PNETs are hyperintense on T2W images and hyper- or isointense during the arterial/pancreatic phase of the dynamic study [6]

In both methods, a big and heterogeneous pancreatic mass (necrotic or hemorrhagic content) are characteristic of NF-PNETs. Multiple hypervascular lesions are frequent in NEM1 [6].

MRI advanced techniques include Diffusion Weighted Image (DWI) and the Apparent Diffusion Coefficient (ADC). DWI is helpful to depict small PNETs due to its greater image contrast. ADC values have been shown to be lower than adjacent pancreatic parenchyma in all cases of solid nodules. However, higher ADC values can be obtained in case of cystic pattern [6]. 
Endoscopic Ultrasonography (EUS) examination is the most sensitive imaging procedure to detect small $(\leq 10 \mathrm{~mm})$ pancreatic endocrine tumors in asymptomatic MEN1 patients; its sensitivity is higher than $75 \%$. The use of EUS in association with Octreoscan scintigraphy (SRS) increases the pancreatic tumoural detection rate to $90 \%$. EUS allows precise localization of the tumors, while SRS gives much more information about the spread of disease and detects liver metastases with a sensitivity of $92 \%$ [7].

In 2015, van Asselt S. J. and colleagues published a study showing that EUS had the biggest sensibility for detection of pancreatic lesions, being superior to CT/MRI plus SRS. With conventional screening, $\geq 1$ pancreatic lesions were detected by CT or MRI in 14 patients $(34 \%)$, by SRS in 12 patients $(29 \%)$, and by CT or MRI plus SRS in 18 patients $(44 \%)$. With EUS, 101 pancreatic lesions were detected in 34 patients $(83 \%)$ with a mean size of 9.1 $+/-7.5 \mathrm{~mm}$.

Different lesions characteristics were identified. Most lesions were homogeneous, hypoechoic, and isoelastic, and $42 \%$ of lesions were hypervascular. Of all lesions, 15 were cystic $(15 \%)$; 9 lesions had thickened walls, which were hypervascular in 5 lesions [8].

Since PNETs are increasingly being recognized, with recent studies indicating these tumors as the most common enteropancreatic NET associated to MEN1 and, furthermore, associated to a worse prognosis than other functioning tumors including insulinoma and gastrinoma, and indicating also that malignant PNETs are reported to be the commonest cause of death in individuals with MEN1, 2012 guideline recommends annual biochemical and image screening, with the methods above explained, to patients with family history of $M E N 1$ or in other individuals with high risk of developing the syndrome, to begin at 10 years old [1].

Also, it emphasizes that PET/CT with DOTATOC/Ga68 may be restricted to doubtful cases, in whom other methods cannot be elucidative [1].

Concerning about treatment, the management of asymptomatic nonfunctioning pancreatic NET is controversial. The goal of treatment is to reduce morbidity and mortality associated with metastatic disease while preserving pancreatic tissue and avoiding complications associated with surgery.

The pancreatoduodenal surgery may cause some complications as Diabetes Mellitus and frequent steatorrhea, but accordingly to some data, the tumor excision may be successful in $80 \%$ of patients.

The 2012 Guideline suggests considering surgical resection for nonfunctioning pancreatic NET that are more than $1 \mathrm{~cm}$ in size. Long-term cure can only be achieved by pancreatectomy. For tumors less than $1 \mathrm{~cm}$ in size, they suggest surgical resection for those that have significant growth, such as a doubling of tumor size, over a 3- to 6-month interval and exceed $1 \mathrm{~cm}$ in size. Other current practice references recommend the surgical removal of functioning PNET and non-functioning PNET exceeding $10-20 \mathrm{~mm}$. However, in view of the potential morbidity of surgery, this approach requires an informed patient choice. It emphasizes also that, accordingly to some studies, there is a number of patients that can present with occult metastatic disease (the metastases doesn't appear in image studies but can be responsible for disease recurrence). There has been a recommendation toward a less aggressive indication of surgery during the past years, and it could be shown in a large multicentric retrospective series that PNETs $<20 \mathrm{~mm}$ pose a low oncological risk $[1,10]$.

In 2006, Triponez et. Al. published a study comparing patients with PNETs less than $2 \mathrm{~cm}$ in two groups: early surgery versus conservative approach. Their data showed no difference between tumor progression in both groups, and also patients with conservative approach had the same life expectancy that MEN1 patients without PNETs. Thus, in their center, surgery isn't recommended to patients with PNETs measuring less than $2 \mathrm{~cm}$ of size [9].

\section{Conclusions}

Neuroendocrine tumors are rare neoplasms, but commonly related to $M E N \quad 1$ Syndrome. The main findings in CT and MRI are multiple, hypervascularized lesions, presenting with a heterogeneous aspect, sometimes with a hemorrhagic/ necrotic component associated.

In $M E N 1$, the most frequent tumors are the Parathyroid Adenomas, which can cause primary hyperparathyroidism, and patients may present with hypercalcemia, nephrolithiasis, and osteitis fibrosa cystica. In second place comes the Pancreatic Neuroendocrine Tumors (PNETs) and the less common are the Pituitary Tumors.

Endoscopic Ultrasound is the most sensible method to diagnose PNETs related to NEM1 syndrome and when associated with scintigraphy (SRS), its sensitivity is even greater.

Scintigraphy with somatostatin analog has high sensitivity in finding metastasis in other anatomic sites, even though the main site is the liver.

The American Endocrine Society in a 2012 guideline suggests beginning at the age of 10 years the screening of patients with a high risk of developing the syndrome. This screen must be with biochemical and image techniques. It also recommends PET/CT with DOTATOC/Ga68 to be restricted to cases in which other image methods aren't elucidative.

Treatment must be done with surgery in patients with PNETs over $1 \mathrm{~cm}$ of size (accordingly to the Endocrine Society), but in some centers, it is recommended to be over $2 \mathrm{~cm}$, as the evidence in this centers showed no benefit of surgery in smaller tumors. Tumor excision may be successful in $80 \%$ of patients, but it can cause some complications, mainly Diabetes. 
Treatment to Parathyroid Adenomas is typically surgical, with removal of the overactive glands with either a subtotal or total parathyroidectomy. Persistent hypercalcemia may be a considerable side effect of surgery.

\section{Acknowledgements}

We disclose no conflicts of interests

\section{REFERENCES}

[1] Thakker R V., Newey PJ, Walls G V., Bilezikian J, Dralle H, Ebeling PR, et al. Clinical practice guidelines for multiple endocrine neoplasia type 1 (MEN1). J Clin Endocrinol Metab. 2012; 97(9):2990-3011.

[2] Amin S, Kim MK. Islet Cell Tumors of the Pancreas. Gastroenterol Clin North Am [Internet]. 2016; 45(1):83100.

[3] Carroll RW. Multiple endocrine neoplasia type 1 (MEN1). Asia-Pac J Clin Oncol. 2013; 9:297-309.

[4] Keller HR, Record JL, Lall NU. Multiple Endocrine Neoplasia Type 1: A Case Report With Review of Imaging Findings. Ochsner J. 2018 Summer; 18(2): 170-175.

[5] Cloyd JM, Poultsides GA. Non-functional neuroendocrine tumors of the pancreas: Advances in diagnosis and management. World J Gastroenterol. 2015; 21(32):9512-25.

[6] Dromain C, Déandréis D, Scoazec JY, Goere D, Ducreux M, Baudin E, et al. Imaging of neuroendocrine tumors of the pancreas. Diagn Interv Imaging [Internet]. 2016; 97(12):1241-57.

[7] Marini F, Falchetti A, Monte F Del, Sala SC, Gozzini A, Luzi E, et al. Multiple endocrine neoplasia type 1. Orphanet J Rare Dis. 2006; 1(1):1-9.

[8] Asselt SJ Van, Brouwers AH, Dullemen HM Van, Pet C--htp. EUS is superior for detection of pancreatic lesions compared with standard imaging in patients with multiple endocrine neoplasia type 1. Gastrointest Endosc. 2015; 81(1).

[9] Triponez F, Goudet P, Dosseh D, Cougard P, Bauters C, Murat A, Cadiot G, Niccoli-Sire P, Calender A, Proye CA 2006 Is surgery beneficial for MEN1 patients with small $(\leq 2$ $\mathrm{cm})$, nonfunctioning pancreaticoduodenal endocrine tumor? An analysis of 65 patients from the GTE. World J Surg 30:654-662; discussion 663-664.

[10] Albers MB, Manoharan J, Bollmann C, Chlosta MP, Holzer K, \& Bartsch DK. Results of Duodenopancreatic Reoperations in Multiple Endocrine Neoplasia Type 1. World Journal of Surgery. 2018. 